\title{
Bladder Pain Syndrome: A Review
}

Authors:

Disclosure:

Received:

Accepted:

Keywords:

Citation:
*Gokhan Calik, Jean de la Rosette

Istanbul Medipol University, Faculty of Medicine, Department of Urology, Istanbul, Turkey

*Correspondence to drgokhan80@hotmail.com

The authors have declared no conflicts of interest.

08.01 .2020

09.03 .2020

Bladder pain syndrome (BPS), empirical, visceral pain syndromes.

EMJ Urol. 2020;8[1]:38-45

\section{Abstract}

Therapy of bladder pain syndrome (BPS) presents a significant challenge in clinical practice. Over the last 20 years, there have been important efforts directed at understanding the syndrome's aetiology and therapeutic challenges. Data regarding disease progression, remission, and prevention are very limited and little is known about the risk factors for the development of associated symptoms over time. Several visceral pain syndromes and systemic diseases often occur together in the same patient. Patients are currently treated by different clinicians on an empirical basis with a variety of different medications and other treatment interventions. Treatment approaches are local or systemic and range from behavioural, to pharmacological, and finally to surgical, which altogether are focussed on optimising quality of life. Treatment of BPS often requires a trial and error approach. The aim of this review is to analyse and present contemporary literature regarding BPS.

\section{INTRODUCTION}

Bladder pain syndrome (BPS) is a chronic bladder pain condition presenting in patients without any identifiable cause. This terminology is used instead of painful bladder syndrome, pelvic pain, or the most commonly used interstitial cystitis. Interstitial cystitis is not an appropriate term because research has shown that bladder inflammation (cystitis) is not clearly involved in the pathophysiology and the abnormal interstitium of the bladder is not reliably related to the disease.

The Society for Urodynamics and Female Urology (SUFU) defines this syndrome as "an unpleasant sensation (pain, pressure, discomfort) perceived to be related to the urinary bladder, associated with lower urinary tract symptoms of more than 6 weeks duration, in the absence of identifiable causes." Definitions that require longer duration of symptoms (e.g., 6 months) should not be taken into consideration because the prevention of withholding treatment in BPS patients is clinically important. Also, definitions from research studies should be omitted during the clinical practice as these may cause misdiagnosis or delayed diagnosis.

Vague characteristics of the disease have led studies to underestimate its prevalence, but it is generally accepted as a female-dominant disorder. ${ }^{2}$ The diagnosis is generally made after the fourth decade of life but may be delayed depending on the clinical suspicion. ${ }^{3}$ Prevalence rates vary greatly; in the NHANES III survey from the USA, the prevalence was recored as 470 per 100,000 population, which was 60 per 100,000 
men and 850 per 100,000 women. ${ }^{4}$ These numbers indicate that nearly 83,000 men and 1.2 million women have BPS in the USA. In Europe, prevalence figures are much lower: 8-16 per 100,000 women. ${ }^{5}$ The patient's age should not be an exclusion criterion, as BPS is observed amongst children and adolescents. ${ }^{6}$

BPS should be a diagnosis of exclusion. The RAND Interstitial Cystitis Epidemiology (RICE) study investigating women with chronic bladder pain used exclusion criteria. Patients with bladder cancer, urethral diverticulum, spinal cord injury, stroke, Parkinson's disease, multiple sclerosis, spina bifida, cyclophosphamide treatment, radiation treatment to the pelvic area, tuberculosis affecting the bladder, uterine cancer, ovarian cancer, vaginal cancer, genital herpes, and pregnancy are all excluded. ${ }^{7}$ During the diagnosis, similar exclusion criteria should be adopted for men also. In the RICE study, two definitions had been established for women based on either high sensitivity or specificity. The high sensitivity definition dictates pain, pressure, or discomfort in the pelvic area, alongside a daytime urinary frequency greater than 10 to avoid the discomfort and to be without fear of wetting. The high specificity definition includes two more criteria, specifically the presence of antibiotic treatment-refractory symptoms and absence of hormonal injections for endometriosis in women. High sensitivity criteria correctly identify BPS patients in $81 \%$ of cases with $54 \%$ specificity, whereas high specificity criteria correctly exclude non-BPS cases in $83 \%$ of cases with $48 \%$ sensitivity. ${ }^{7-9}$

Most patients with BPS feel pain or discomfort while holding urine and take relief after micturition. ${ }^{10}$ It is important to define pain in a broad spectrum because some patients may deny pain but instead report pressure feeling.

In addition to pain, urgency, frequency, nocturia, dysuria, dyspareunia, bladder spasms, and suprapubic pressure sensation are common symptoms. ${ }^{11}$ Patients usually define an increasing number of symptoms after the single initial symptom, such as dysuria, frequency, or pain. Symptoms vary from time to time and severity also changes. The onset of symptoms is generally gradual but some patients may define an abrupt onset without a triggering situation. Sudden intensifying symptom flares sometimes occur and may continue for several hours, days, or weeks. Symptoms may exacerbate during stress, menstruation, exercise, sexual intercourse, prolonged sitting, or following intake of certain foods or drinks. ${ }^{12-14}$ Patients tend to hold their urine volume as low as possible and try to empty their bladder at the first opportunity; despite this, urinary incontinence is rarely observed. A possible aetiology may be related to bladder wall problems. Bladder impermeability to solutes stems from the glycosaminoglycan layer of the urothelial surface and defects in this impermeable surface expose the urothelium to urinary irritants, in turn leading to tissue damage, hypersensitivity, and pain. ${ }^{15}$

On physical examination, tenderness in the hip girdle, abdominal wall, urethra, and pelvic floor can be felt, as well as potential accompaning scrotal and penile tenderness in men. Significant pelvic prolapse, inguinal hernia, urethral diverticulum, uterine or cervical mass, prostate induration, and eroded or exposed vaginal mesh should be checked during the examination.

Impact on psychosocial functioning and quality of life is very typical in patients with BPS. BPS may cause depression, anxiety, loss of worklife balance, stress, panic disorder, and sexual dysfunction. ${ }^{16}$ BPS patients may be reactive for these mental health situations, but there is also some evidence suggesting a genetic link between BPS and panic disorder. ${ }^{17}$ There may be comorbidities associated with BPS, such as fibromyalgia, irritable bowel syndrome, chronic fatigue syndrome, Sjögren's syndrome, chronic headaches, and vulvodynia, 18 and common pathophysiology may exist. BPS may be part of a more generalised systemic disorder. Also, additional epidemiological studies suggest that many of these somatic symptoms are also present if the BPS patient is correctly assessed during the diagnostic workup. In addition to this, another hypothesis is that BPS may be a part of the continuum of painful versus non-painful overactive bladder syndrome. ${ }^{19}$

Quality of life is lower when compared to the controls; 20 because of this, social, educational, and career-related activities may not be pursued. Additionally, emotional distress, depression, and social isolation may be substantial. 
There is an increased interest in BPS among the urology community because of the issue pertaining to quality of life and genitourinary function. The aim of this article is to provide contemporary knowledge to the general urologist.

\section{ASSESSMENT}

\section{Costs}

The economic burden of BPS on the healthcare system is immense. Direct costs associated with BPS include physician visits, prescription medications, outpatient procedures, and hospitalisation. These costs are greater than the mean annual per person direct costs of diabetes, depression, hypertension, and asthma. ${ }^{21}$ Because of the chronic nature of the disease, these costs typically persist for years. Indirect costs of BPS include lost productivity while working or time away from work. This is a serious problem because the condition primarily affects those of working age (i.e., 25-50 years old).

\section{Patient History}

A history of pelvic pain irrelative with bladder filling, haematuria, severe urinary incontinence, prior surgery, pelvic trauma, recurrent urinary tract infections, irradiation, or neurological disorder may prompt for diagnostic cystoscopy and/or urodynamics. A 2-day voiding chart is recommended for all BPS patients. A frequent and low volume $(<300 \mathrm{~mL})$ voiding pattern is generally observed.

\section{Urinary Tests and Instrumentation}

Standard urine tests and post-void urine should be checked in all patients. A urine culture should be added if the urine test indicated an infection. Routine cystoscopy is not recommended but in the event of clinical suspicion for various situations (e.g., haematuria, unresponsiveness to previous treatment) it should be performed. There are two findings during cystoscopy: Hunner lesions, which are reddened lesions on the bladder mucosa with attached fibrin deposits that typically bleed after hydrodistention; and glomerulations, which are petechial red areas seen during the cystoscopy. In BPS patients these are of limited diagnostic value because they are nonspecific findings (e.g., one study found glomerulations in $45 \%$ of healthy patients), and their presence does not correlate well with symptoms. ${ }^{22,23}$ An increased number of mast cells may be present on bladder biopsies.

\section{Additional Tests}

The potassium sensitivity test is best avoided because of the extreme pain felt during the procedure and the result not being specific to BPS. ${ }^{24}$ Instillation of lidocaine to the bladder is also not reliable.

\section{TREATMENT}

As BPS is a chronic disorder, after the initial diagnosis all patients must be informed about the unavailability of curative treatment and that the goal is to provide relief of symptoms to achieve a good quality of life. Some studies suggest that BPS has a waning and waxing course with an average improvement over time, but other papers report patients with more improvement. ${ }^{25,26}$ These conflicting results from different studies are not surprising because they were produced from different patient populations with varying purposes.

In BPS, initial measures applied to the patients should be patient education, treatment of comorbid diseases, and psychosocial support. The treatment strategy should start from the most appropriate conservative options and proceed to the less conservative therapies if symptom control is not enough. No single therapy is successful and continuously effective in all patients. ${ }^{27}$ In order to optimise the quality of life, the treatment method should be tailored to the specific symptoms of each patient until the more effective therapies are available. After a reasonable period of the unsuccessful treatment trial, it is justifiable to advance to the next treatment level assuming consensus between the patient and the clinician. In instances where patients have a more complex presentation and/ or have failed response to standard treatment approaches, urologists may need to co-operate with other clinicians such as gynecologists, gastroenterologists, pain specialists, physical therapists, primary care providers, nurse practitioners, and dietitians. Each treatment is individual, and if a patient rapidly develops 
worsening symptoms it is wise to proceed to more aggressive therapies.

The American Urological Association (AUA) has issued clinical practice guidelines for the treatment of interstitial cystitis/BPS. ${ }^{28}$

\section{First-Line Treatments}

Self-care practices and behavioural modification should be discussed with the patient. Patients should be encouraged to cope with stress and manage stress-induced symptom exacerbations. Application of local heat or cold over the perineum or bladder; avoidance of activities, foods, or drinks that exacerbate the complaints; pelvic floor muscle relaxation; bladder training with urge suppression; fluid restriction; or additional hydration to alter the concentration and/or volume of urine can be recommended. ${ }^{27,29}$ Foster et al. ${ }^{30}$ reported that $45 \%$ of 136 BPS patients treated with these measures had a moderate-to-marked improvement at 12 weeks.

Stress increases BPS symptoms and effective coping with family, work, or post-traumatic experience is an important part of the management. ${ }^{31}$ Clinicians should educate the patients about normal bladder function. They should try to explain what is known and not known about BPS and also make short comparisons between the benefits and risks of treatment alternatives. There are two facts that should be emphasised by the clinician: first, that there is no single treatment that is effective for the majority of patients, and second, that symptom control may require trials of various treatment options before seeing marked improvement.

\section{Second-Line Treatments}

Many BPS patients experience pelvic floor muscle tenderness and pain so appropriate manual physical therapy techniques may be applied. The benefits of physical therapy have previously been calculated as a 59\% response rate in receivers versus $29 \%$ in nonreceivers. ${ }^{32}$ Pelvic floor strengthening exercises like Kegel should be avoided because they may worsen the symptoms.

There are no comparative studies of oral medications for interstitial cystitis/BPS; the choice of agent depends upon the risk of adverse effects and patient preference. Tricyclic antidepressants such as amitriptyline are believed to have analgesic properties and to also relieve the depressive symptoms associated with chronic pain. In a clinical randomised trial, oral amitriptyline ( $25 \mathrm{mg}$ daily titrated over several weeks to $100 \mathrm{mg}$ daily if tolerated) was reported to be superior to placebo ( $63 \%$ versus $4 \%$ ) over 4 months. ${ }^{33}$ Sedation, nausea, and drowsiness are extremely common adverse effects (up to $79 \%$ of patients) of amitriptyline therapy.

Pentosan polysulfate sodium (PPS) has a modest benefit in controlling BPS symptoms according to a randomised clinical study. ${ }^{28}$ The proposed mechanism of action of PPS is that it reconstitutes the deficient protective glycosaminoglycan layer over the urothelium. In reality, however, only a tiny proportion of PPS is absorbed by the gastrointestinal tract and excreted in the urine of BPS patients. ${ }^{34}$ Multiple randomised controlled trials have reported final outcomes of symptom reduction ranging from $21 \%$ to $56 \%$ in BPS patients, compared with $13 \%$ to $49 \%$ of patients under placebo. Adverse effects are not significant and seen only in $10-20 \%$ of patients. However, rare serious adverse effects on visual acuities such as metamorphopsia, blurred vision, and prolonged dark adaptation have been reported. PPS-associated maculopathy is a vision-threatening condition that can manifest in the setting of long-term exposure to the drug, and is a situation of ongoing interest in ophthalmology. ${ }^{35}$

Hydroxyzine is the most commonly used antihistamine for the treatment of BPS. The use of antihistamines for BPS is based upon the hypothesis that the pathogenesis process is hypersensitive in this condition. In one study, $23 \%$ of BPS patients treated with oral hydroxyzine (10-50 mg daily titration over several weeks if tolerated) for 6 months experienced symptom relief, however $13 \%$ of patients under placebo also felt symptom relief. This difference was not statistically significant. ${ }^{36}$ Adverse effects (sedation, weakness) are common in up to $82 \%$ of patients but not serious. ${ }^{37}$

Cimetidine is used for its anti-inflammatory effect. Two observational studies have reported an improvement of symptoms in 44-57\% 
of patients at follow-up without significant adverse effects. ${ }^{38,39}$

Dimethyl sulfoxide has a mechanism of action that is thought to be multifactorial, including anti-inflammatory, analgesic, smooth-muscle relaxing, and mast-cell inhibitory effects. ${ }^{40}$ Dimethyl sulfoxide is reserved for patients who have failed oral medications. Its use is limited because it is associated with a shortterm exacerbation of symptoms and requires multiple hospital visits for bladder catheterisation.

Heparin and lidocaine are also second-line bladder instillation agents offering short-term relief and minimal side effects.

\section{Third-Line Treatments}

Cystoscopy under anesthaesia with hydrodistension (6O-8O $\mathrm{cm} \mathrm{H}_{2} \mathrm{O}$ ) for a short duration (10 minutes) is an appropriate technique in patients failing more conservative therapies. Observational studies have reported clinical relief to range from $30 \%$ to $54 \%$ at 1 month, $18 \%$ to $56 \%$ at $2-3$ months, and $0 \%$ to $7 \%$ at 5-6 months. Significant adverse effects were not reported. ${ }^{41-43}$ If Hunner lesions are present during cystoscopy then fulguration treatment should be applied. Post-treatment pain relief in patients is in the range of $75-86 \% .44,45$ There were no serious side effects but the patients should be informed about the re-treatment for when symptoms recur.

\section{Fourth-Line Treatments}

Intradetrusor botulinum toxin Type-A (BTX-A) and neuromodulation are fourth-line therapies for BPS. The mechanism of BTX-A therapy effect for BPS is likely in its ability to modulate sensory neurotransmission. Two studies reported high initial efficacy rates of $74 \%$ and $86 \%$ at 3 months. ${ }^{46,47}$ The treatment effect is temporary and usually diminishes over a year.

The patients who are willing to receive BTX-A treatment should be informed about the potential for chronic urinary retention and also be able to perform self-catheterisation if needed.

The sacral neuromodulation device consists of an implanted lead that lies along a sacral nerve root (usually S3) and is attached to an implanted pulse generator. Alternatively, the lead can be placed to stimulate the pudendal nerve. Observational studies suggest symptomatic relief; however, the rate of re-intervention is high. A small retrospective observational study with an average follow-up of 60 months suggested that treatment of BPS with an implant provides long-term symptomatic relief for most patients; however, this study and another showed a high rate of re-intervention. Reasons for re-intervention included device malfunction, treatment failure, or loss of benefit. 48,49

\section{Fifth-Line Treatments}

The use of oral cyclosporine A has been reported to decrease the symptoms of some BPS patients, especially those with Hunner lesions. This agent is of limited use because of the severity of potential adverse effects, including nephrotoxicity, hypertension, immunosuppression, hair growth, gingival hyperplasia, paresthesias, abdominal pain, flushing, and muscle pain..$^{50} \mathrm{~A}$ randomised trial $(\mathrm{N}=64)$ to evaluate this therapy compared 6 months of treatment with oral cyclosporine A (1.5 mg/kg twice daily) to PPS (100 mg three times daily). Cyclosporine A showed significantly better results for improvement on a symptom scale (75\% versus 19\%) and reduction in urinary frequency (6.7 times versus 2.0 times per 24 hours). ${ }^{51}$

\section{Sixth-Line Treatments}

Treatment-refractory symptomatic patients, those with significant loss of life quality, and those that are motivated to undergo irreversible major surgery are candidates for this lastresort treatment. The informed consent process is critical. Carefully selected patients must understand that pain relief is not guaranteed and pain can persist even if the bladder is removed. Substitution cystoplasty and urinary diversion with or without cystectomy are surgical options for this group of patients. Small bladder capacity under anesthaesia and the absence of neuropathic pain are associated with better outcomes. ${ }^{52,53}$

Figure 1 summarises the treatment algorithm; however, all treatment stages should be tailored to the symptomatology of each individual patient at the discretion of the physician. Following the treatment algorithm step-by-step is not mandatory and some steps may be skipped or reversed during the course of the therapy. 


\section{BPS Patient}

Basic assessment: history, physical exam,post-void residual volume, urinalysis and culture, frequency/volume chart, pain/symptom questionnaires

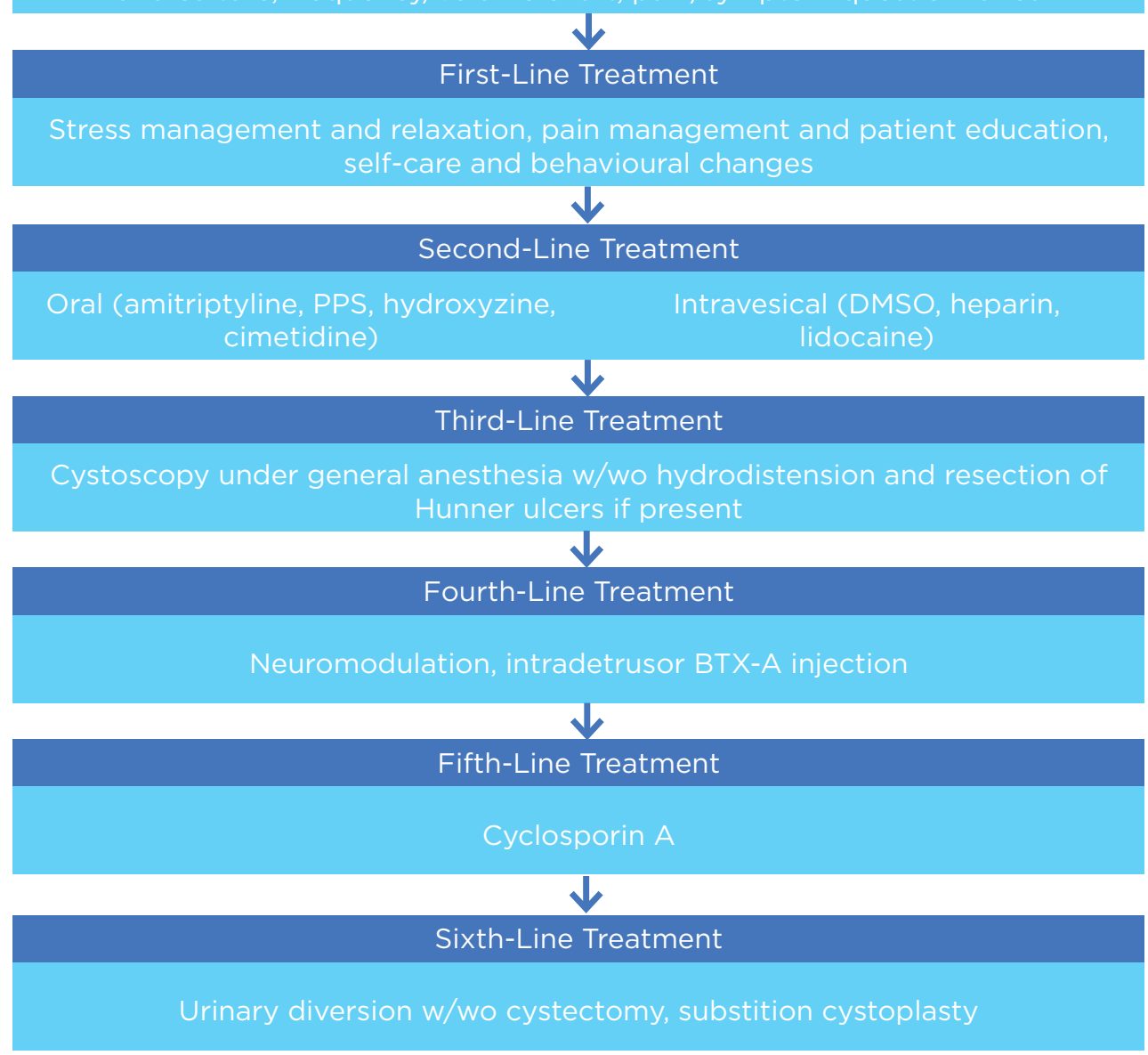

\section{Figure 1: Treatment algorithm for bladder pain syndrome.}

BPS: bladder pain syndrome; BTX-A: botulinum toxin Type-A; DMSO: dimethyl sulfoxide; PPS: pentosan polysulfate sodium; w/wo: with or without.

\section{CONCLUSION}

BPS is defined as an unpleasant sensation (pain, pressure, discomfort) perceived to be related to the urinary bladder, associated with lower urinary tract symptoms of $>6$ weeks' duration, in the absence of identifiable causes. The characteristics of the bladder pain or discomfort in patients with BPS are variable, but the most common is an increase in discomfort with bladder filling and relief with voiding. BPS is a chronic pain syndrome and the aetiology is not well understood. Focus on biomarkers such as APF is the next area of research.
The initial diagnostic evaluation of most patients with BPS may be performed by a primary care physician. However, certain patients with bladder pain symptoms warrant referral to a specialist, such as a urologist or urogynecologist, for additional diagnostic testing. Treatment is not curative and the goal of management is to provide relief of symptoms to achieve an adequate quality of life. There are many therapeutic approaches for BPS and none are proven to be helpful for all patients. BPS is treated with a step-wise approach guided by individual patient characteristics and the risk for adverse effects associated with each type of treatment. 


\section{References}

1. Hanno P, Dmochowski R. Status of international consensus on interstitial cystitis/bladder pain syndrome/ painful bladder syndrome: 2008 snapshot. Neurourol Urodyn. 2009;28(4):274-86.

2. Clemens JQ et al. Interstitial Cystitis and Painful Bladder Syndrome. Litwin MS, Saigal CS (eds.), Urologic Diseases in America (2007), Washington: US Department of Health and Human Services, Public Health Service, National Institutes of Health, National Institute of Diabetes and Digestive and Kidney Diseases, pp.123.

3. Simon LJ et al. The interstitial cystitis data base study: concepts and preliminary baseline descriptive statistics. Urology. 1997;49(5A Suppl):64-75.

4. Payne CK et al. Interstitial cystitis and painful bladder syndrome. J Urol. 2007:177(6):2042-9.

5. Bade JJ et al. Interstitial cystitis in The Netherlands: prevalence, diagnostic criteria, and therapeutic preferences. J Urol. 1995;154(6):2035-7; discussion 2037-8.

6. Mattox TF. Interstitial cystitis in adolescents and children: a review. J Pediatr Adolesc Gynecol. 2004;17(1):7.

7. Berry SH et al. Prevalence of symptoms of bladder pain syndrome/ interstitial cystitis among adult females in the United States. J Urol. 2011;186(2):540-4

8. Berry $\mathrm{SH}$ et al. Development validation and testing of an epidemiological case definition of interstitial cystitis/painful bladder syndrome. J Urol. 2010;183(5):184852.

9. Suskind AM et al. The prevalence and overlap of interstitial cystitis/ bladder pain syndrome and chronic prostatitis/chronic pelvic pain syndrome in men: results of the RAND interstitial cystitis epidemiology male study. J Urol. 2013;189(1):141-5.

10. Bogart LM et al. Symptoms of interstitial cystitis, painful bladder syndrome and similar diseases in women: a systematic review. J Urol. 2007;177(2):450-6.

11. Teichman JM, Parsons CL.

Contemporary clinical presentation of interstitial cystitis. Urology. 2007;69(4 Suppl):41-7.

12. Friedlander JI et al. Diet and its role in interstitial cystitis/bladder pain syndrome (IC/BPS) and comorbid conditions. BJU Int. 2012;109(11):158491.

13. Rothrock NE et al. Stress and symptoms in patients with interstitial cystitis: a life stress model. Urology. 2001;57(3):422-7.
14. Powell-Boone T et al. Menstrual cycle affects bladder pain sensation in subjects with interstitial cystitis. J Urol. 2005;174(5):1832-6.

15. Parsons $\mathrm{CL}$. The role of the urinary epithelium in the pathogenesis of interstitial cystitis/prostatitis/ urethritis. Urology. 2007;69(4):9-16.

16. Clemens JQ et al. Prevalence of painful bladder symptoms and effect on quality of life in black, Hispanic and white men and women. J Urol. 2007;177(4):1390-4

17. Weissman MM et al. Interstitial cystitis and panic disorder: a potential genetic syndrome. Arch Gen Psychiatry. 2004;61(3):273-9.

18. Clemens JQ et al. Case-control study of medical comorbidities in women with interstitial cystitis. J Urol. 2008;179(6):2222.

19. Philip $\mathrm{J}$ et al. Interstitial cystitis versus detrusor overactivity: a comparative, randomized, controlled study of cystometry using saline and $0.3 \mathrm{M}$ potassium chloride. J Urol 2006;175(2):566-70; discussion 570-1.

20. Rothrock NE et al. Depressive symptoms and quality of life in patients with interstitial cystitis. J Urol. 2002;167(4):1763-7.

21. Clemens JQ et al. Costs of interstitial cystitis in a managed care population. Urology. 2008;71(5):776-80.

22. Parsons $C L$ et al. The role of urinary potassium in the pathogenesis and diagnosis of interstitial cystitis. J Urol. 1998;159(6):1862-7.

23. Tomaszewski JE et al. Biopsy features are associated with primary symptoms in interstitial cystitis: results from the interstitial cystitis database study. Urology. 2001;57(6 Suppl 1):67-81.

24. Hanno P. Is the potassium sensitivity test a valid and useful test for the diagnosis of interstitial cystitis? Int Urogynecol J. 2005;16:428-9.

25. Link CL et al. Prevalence and psychosocial correlates of symptoms suggestive of painful bladder syndrome: results from the Boston area community health survey. J Urol. 2008;180(2):599-606

26. Propert KJ et al. A prospective study of interstitial cystitis: results of longitudinal followup of the interstitial cystitis data base cohort. J Urol. 2000:163(5):1434-9.

27. Rovner $E$ et al. Treatments used in women with interstitial cystitis: the interstitial cystitis data base (ICDB) study experience. Urology. 2000;56(6):940-5

28. Hanno PM et al. Diagnosis and treatment of interstitial cystitis/ bladder pain syndrome: AUA guideline amendment. J Urol.
2015;193(5):1545-53.

29. Shorter B et al. Effect of comestibles on symptoms of interstitial cystitis. $J$ Urol. 2007;178(1):145-52.

30. Foster HE et al. Effect of amitriptyline on symptoms in treatment naïve patients with interstitial cystitis/ painful bladder syndrome. J Urol 2010;183(5):1853-8.

31. Lutgendorf SK et al. Stress and symptomatology in patients with interstitial cystitis: a laboratory stress model. J Urol. 2000;164(4):1265-9.

32. FitzGerald MP et al. Randomized multicenter clinical trial of myofascial physical therapy in women with interstitial cystitis/painful bladder syndrome and pelvic floor tenderness. J Urol. 2012;187(6):2113-8.

33. van Ophoven A et al. A prospective, randomized, placebo controlled, double-blind study of amitriptyline for the treatment of interstitial cystitis. J Urol. 2004;172(2):533-6.

34. Erickson DR et al. Molecular size affects urine excretion of pentosan polysulfate. J Urol. 2006;175(3):1143-7.

35. Hanif AM et al. Phenotypic spectrum of pentosan polysulfate sodiumassociated maculopathy: a multicenter study. JAMA Ophthalmol. 2019;137(11):1275-82.

36. Sant GR et al. A pilot clinical trial of oral pentosan polysulfate and oral hydroxyzine in patients with interstitial cystitis. cJ Urol. 2003;170(3):810-5.

37. Theoharides TC. Hydroxyzine in the treatment of interstitial cystitis. Urol Clin North Am. 1994;21(1):113-9.

38. Dasgupta $P$ et al. Cimetidine in painful bladder syndrome: a histopathological study. BJU Int. 2001;88(3):183-6.

39. Seshadri $P$ et al. Cimetidine in the treatment of interstitial cystitis. Urology. 1994;44(4):614-6.

40. Yamada $T$ et al. Adjuvant hydrodistension under epidural anesthesia for interstitial cystitis. Int J Urol. 2003;10(9):463-8.

41. Cole EE et al. Are patient symptoms predictive of the diagnostic and/or therapeutic value of hydrodistention? Neurourol Urodyn. 2005;24(7):63842.

42. Erickson DR et al. Changes in urine markers and symptoms after bladder distention for interstitial cystitis. $J$ Urol. 2007;177(2):556-60

43. Ottem DP, Teichman JM. What is the value of cystoscopy with hydrodistension for interstitial cystitis? Urology. 2005;66(3):494-9.

44. Malloy TR, Shanberg AM. Laser therapy for interstitial cystitis. Uro Clin North Am. 1994;21(1):141-4. 
45. Payne RA et al. Endoscopic ablation of Hunner's lesions in interstitial cystitis patients. Can Urol Assoc J. 2009;3(6):473-7.

46. Liu HT, Kuo HC. Intravesical botulinum toxin $\mathrm{A}$ injections plus hydrodistension can reduce nerve growth factor production and control bladder pain in interstitial cystitis. Urology. 2007;70(3):463-8.

47. Giannantoni A et al. Botulinum A toxin intravesical injections in the treatment of painful bladder syndrome: a pilot study. Eur Urol. 2006;49(4):704-9.
48. Powell CR, Kreder KJ. Long-term outcomes of urgency-frequency syndrome due to painful bladder syndrome treated with sacral neuromodulation and analysis of failures. J Urol. 2010;183(1):173-6.

49. Chughtai B et al. Failures of Sacral Neuromodulation for Incontinence. JAMA Surg. 2018;153:493.

50. Sairanen J et al. Long-term outcome of patients with interstitial cystitis treated with low dose cyclosporine A J Urol. 2004;171(6 Part 1):2138-41.

51. Sairanen J et al. Cyclosporine A and pentosan polysulfate sodium for the treatment of interstitial cystitis: a randomized comparative study. J Urol. 2005;174(6):2235-8.

52. Linn JF et al. Treatment of interstitial cystitis: comparison of subtrigonal and supratrigonal cystectomy combined with orthotopic bladder substitution. J Urol. 1998;159(3):774-8.

53. Lotenfoe RR et al. Absence of neuropathic pelvic pain and favorable psychological profile in the surgical selection of patients with disabling interstitial cystitis. J Urol. 1995:154(6):2039-42. 